\title{
Turning up the heat (shock)
}

Duchenne muscular dystrophy (DMD) is a debilitating genetic disorder in which muscles gradually degenerate and for which there are no effective treatments. Now, a study in Nature suggests that increasing the expression of heat shock protein 72 (HSP72) with the nicotinic amidoxime derivative BGP-15 could be a potential new treatment strategy.

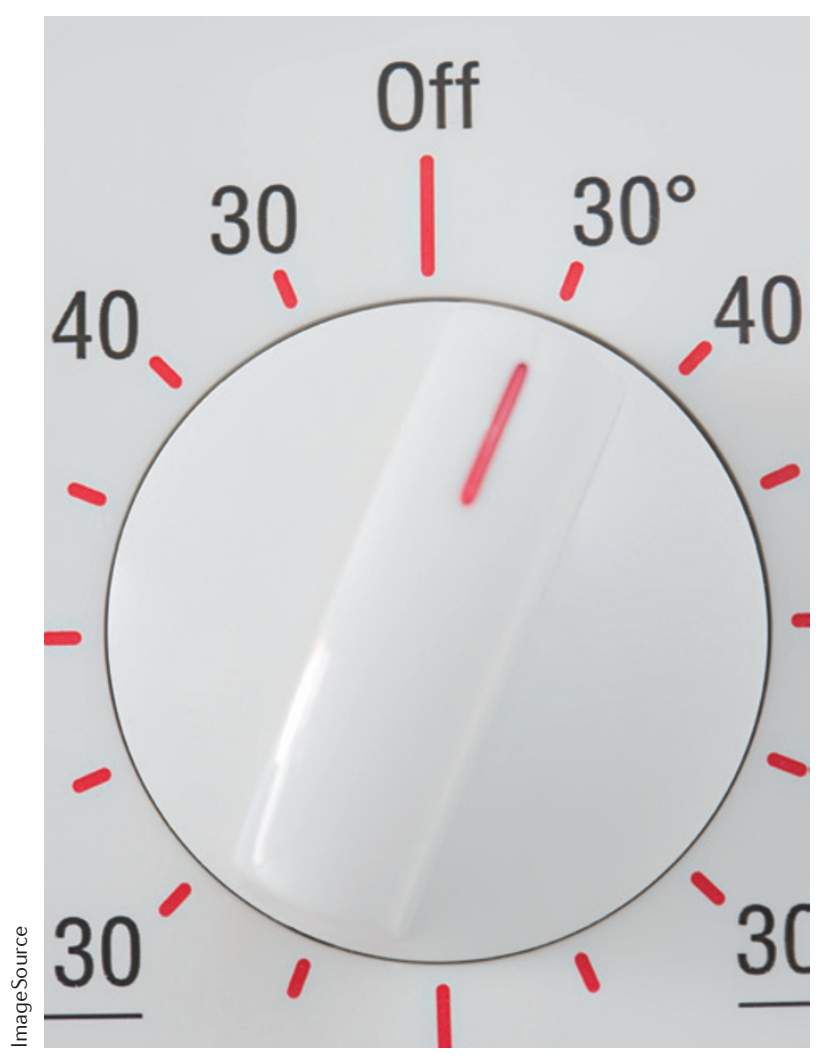

Mutations in the gene encoding the vital cytoskeletal protein dystrophin lead to its deficiency in patients with DMD, and dystrophin-deficient muscles are vulnerable to influxes in $\mathrm{Ca}^{2+}$, which can trigger inflammatory and muscle-degenerative pathways. HSP72 is known to inhibit some of the mediators of the inflammatory response, and so the authors of this study speculated that increasing the levels of this protein could protect dystrophic muscles from degeneration.

First, preliminary studies confirmed the beneficial effects of HSP72 overexpression in dystrophindeficient muscle. Dystrophin-null $(m d x)$ mice crossed with transgenic mice overexpressing muscle-specific HSP72 produced $m d x^{\mathrm{TG}(+)}$ mice, and these progeny had improved wholebody muscle strength, decreased levels of creatine kinase (an indicator of muscle breakdown) and preserved diaphragm function. The latter result is particularly important, as respiratory failure is a major cause of death in patients with DMD.

Next, the authors showed that the function of sarco/endoplasmic reticulum $\mathrm{Ca}^{2+}$-ATPase (SERCA; which has a vital role in $\mathrm{Ca}^{2+}$ homeostasis in muscle) deteriorates in an age-related manner in $m d x$ mice. This effect was ameliorated in $m d x^{\mathrm{TG}(+)}$ mice, in which SERCA activity was preserved. Normally, HSP72 binds SERCA to prevent inactivation under conditions of stress, and the authors suggest that this is probably one of the contributing molecular mechanisms by which HSP72 overexpression protects against muscle degeneration in $m d x^{\mathrm{TG}(+)}$ mice.

As a result of these findings, the authors then investigated whether the effects could be pharmacologically induced, and used BGP-15, which is a known co-inducer of HSP72. $m d x$ mice receiving this agent had increased HSP72 levels, reduced creatine kinase levels and improved diaphragm pathology. Maximal SERCA activity was also observed after long-term (12 weeks) treatment. Importantly, when BGP-15 was given to mice deficient in both utrophin and dystrophin (the most accurate mouse model of the phenotype of DMD), lifelong treatment was able to extend survival.

Together, these results suggest that increasing HSP72 expression in dystrophic muscles is a novel strategy to improve symptoms in DMD, and BGP-15 (which is currently in Phase II clinical trials for diabetes, and has previously been shown to be clinically safe and well tolerated) could be a promising drug candidate to enact these effects.

Man Tsuey Tse

ORIGINAL RESEARCH PAPER Gehrig, S. M. et al.

Hsp72 preserves muscle function and slows progression of severe muscular dystrophy. Nature 4 Apr 2012 (doi:10.1038/nature10980) 